\title{
The yin and yang of retinoic acid signaling in kidney diseases
}

\author{
Qingqing Wei' and Zheng Dong ${ }^{1,2}$ \\ 'Department of Cellular Biology and Anatomy, Medical College of Georgia at Augusta University, Augusta, Georgia, USA. ${ }^{2}$ Charlie Norwood VA Medical Center, Augusta, Georgia, USA.
}

\begin{abstract}
Retinoic acid (RA) signaling is involved in various physiological and pathological conditions, including development, tumorigenesis, inflammation, and tissue damage and repair. In kidneys, the beneficial effect of RA has been reported in multiple disease models, such as glomerulosclerosis, renal fibrosis, and acute kidney injury. In this issue of the $J C l$, Chen et al. report a pathway activated by RA signaling that is mediated by the retinoic acid receptor responder protein 1 (RARRES1). Specifically, RARRES1, which is proteolytically cleaved to release the extracellular domain, was endocytosed by podocytes to induce apoptosis and glomerular dysfunction kidney disease. These findings unveil the contrasting aspects, a Janus face, of RA signaling that may guide its therapeutic use.
\end{abstract}

\section{RA signaling}

Retinoic acid (RA) is the main derivative of vitamin A. After entering the cell, RA binds and activates the nuclear receptors retinoic acid receptor (RAR) and retinoic $\mathrm{X}$ receptor (RXR). The activated ligand/receptor complex enters the nucleus and binds to a specific DNA sequence for the regulation of target gene expression (Figure 1). The RA signaling pathway contributes to various biological processes, ranging from development and physiology to diseases (1-4).

In kidneys, in addition to its indispensable role in renal development (5), RA signaling is beneficial in various kidney diseases, acting by inducing protective genes or attenuating injurious factors (Figure 1). RA signaling is repressed in adult kidneys, but it is reactivated following acute kidney injury (AKI), probably to aid with repair and regeneration (6). Indeed, RA ameliorates ischemic and nephrotoxic AKI by reducing renal tubular cell death and interstitial inflammation (6-10). In AKI, RA suppressed the expression of injuri- ous factors, such as Nur77 and TNF- $\alpha$ (6, 10). In contrast, RA signaling is impaired in chronic kidney disease and the impairment is associated with the appearance of pathological hallmarks, such as renal fibrosis, inflammation, and glomerulosclerosis (11-13). Treatment with RA or RA signaling agonists to reverse this impairment can preserve functional kidney tissues, including tubular cells, mesangial cells, and podocytes $(11,12,14,15)$. These beneficial effects may be associated with the induction of Kruppel-like factor 15 (KLF15) and the reduction of BMP4, iNOS, and TGF- $\beta 1$ (15-18). Notably, the protective mechanism of RA signaling has been examined in depth in podocytes in experimental models of focal segmental glomerulosclerosis (FSGS) and diabetic kidney disease (DKD). It has been shown that albumin overload due to proteinuria can neutralize RA to inactivate RA signaling, leading to the suppression of podocyte regeneration and differentiation (13). RA treatment as well as overexpression of

Related Article: p. 5523

Conflict of interest: The authors have declared that no conflict of interest exists.

RAR or retinol dehydrogenase 9 activates RA signaling and results in gene transcription $(12,14,19)$. RA signaling could further activate the cAMP/cAMP-response element (CREB) pathway, leading to the expression of KLF15 for podocyte differentiation and protection $(16,20)$.

\section{RA signaling in podocytes in glomerular disease}

Despite the documented beneficial effects of RA signaling, the clinical use of RA has raised concerns about its side effects (2123); however, the underlying mechanism remains largely unknown. In this issue of the JCI, Chen and colleagues have identified retinoic acid receptor responder protein 1 (RARRES1) as a key factor mediating the detrimental effect of RA signaling in podocytes in glomerular disease (24). This study started from the analysis of the Nephrotic Syndrome Study Network (NEPTUNE), a transcriptomic data set of human glomerular disease, in which RARRES1 expression showed a positive correlation with the decline of renal function. RARRES1 upregulation was also obvious in the glomeruli of DKD patients in the Nephroseq transcriptomic data set of kidney disease and in the recently published single-cell sequencing study (24). In cultured podocytes, both RA and TNF- $\alpha$ could induce RARRES1, suggesting that TNF- $\alpha$ may stimulate RARRES1 in glomerular disease. Overexpression of RARRES1 potently induced podocyte apoptosis in vitro and glomerular injury in vivo, whereas knockdown of RARRES1 had the opposite effect, indicating the role of RARRES1 in the development and progression of glomerular disease. These observations provide insight into the mechanism of the detrimental effects of RA treatment in clinical settings. Certainly, RARRES1 may not account for all side effects of RA because podocytes are restricted to the glomeruli in kidneys and it is unclear whether RARRES1 is expressed by other cells and in other tissues or organs. Nonetheless, the 


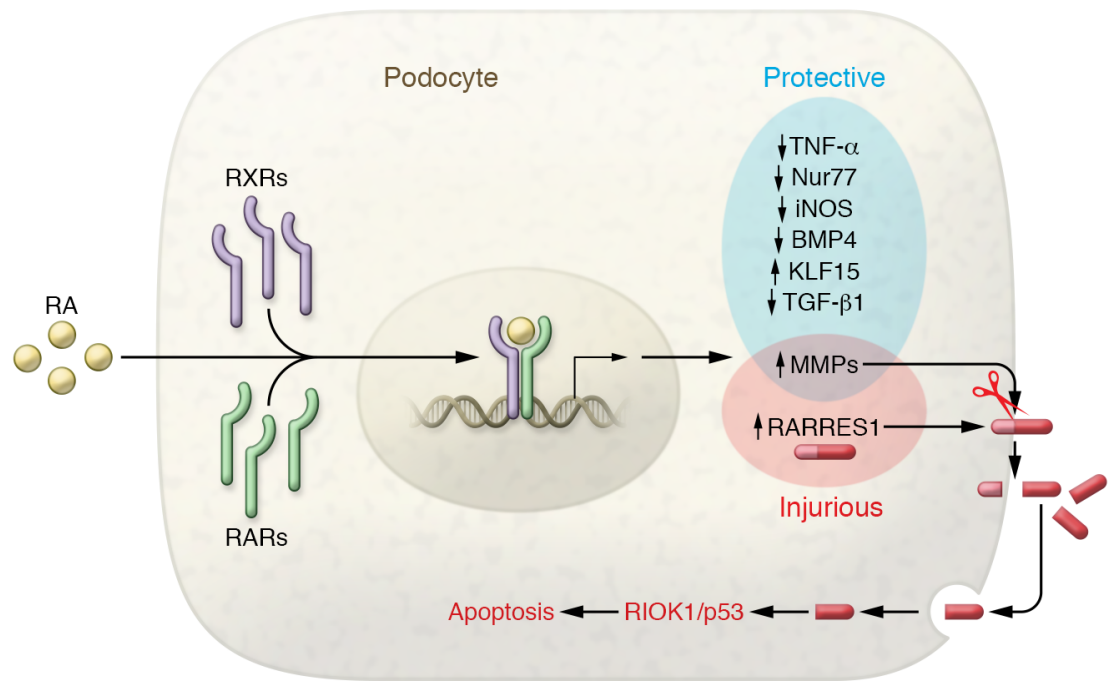

Figure 1. The yin and yang of RA signaling in podocyte injury and protection. RA binds to and activates nuclear receptors RAR/RXR, resulting in expression changes in protective or injurious genes. RARRES1, a gene induced by RA signaling, is cleaved by MMPs to release its C-terminal fragment, which is then endocytosed to activate the RIOK1/p53 apoptosis pathway in podocytes.

work by Chen et al. suggests that blocking RARRES1 would limit the side effects of RA and improve the therapeutic benefits of RA for kidney or glomerular disease.

How does RARRES1 trigger podocyte injury? Chen et al. (24) demonstrated the proteolytic processing of RARRES1, which results in the release of its C-terminal, extracellular domain. Interestingly, the released fragment is endocytosed by podocytes where it may bind to and antagonize RIOK1, a serine/threonine-specific protein kinase with antiapoptotic activities, leading to 553 activation and apoptosis (Figure 1). These findings are fundamental to the understanding of the molecular mechanism whereby RARRES1 induces podocyte injury and glomerular disease. Remarkably, mutation of the cleavage site in RARRES1 diminished the detrimental effects of RARRES1 overexpressed in mice, supporting a critical role of the proteolysis of RARRES1 in its pathogenic effect. Chen et al. also suggested the involvement of metalloproteinases (MMP) in the cleavage of RARRES1 in podocyte injury. While the identity of the responsible MMP remains elusive, MMP2 and MMP9 were induced, along with RAR $\alpha$, in podocytes in an experimental model of FSGS (19). Thus, in glomerular disease, the simultaneous induction of MMPs and RARRES1 by RA may activate a cell death pathway in podocytes, contributing to the side effects of RA treatment. Whether specific inhibitors of MMPs can attenuate RARRES1-mediated podocyte injury and improve the therapeutic effect of RA remains unknown and warrants further investigation.

\section{Perspectives and conclusions}

The crosstalk between different cells in kidneys is crucial to the development of renal diseases, including glomerulosclerosis, which may involve glomerular cells as well as extraglomerular cells (25). The Chen et al. study provides insights into RARRES1 function and regulation, which includes MMP-mediated proteolysis, consequent release of its C-terminal fragment, endocytosis of the fragment by podocytes, and the activation of RIOK1/p53 signaling for cell death (Figure 1). Although this study only examined autocrine action on podocytes, the RARRES1 fragment released into the extracellular space may affect other cells in kidneys as well, especially the adjacent mesangial cells, parietal epithelial cells, and capillary endothelial cells in glomeruli. In addition, the RARRES1 fragment may leak into tubular lumen to affect renal tubular cells. Thus, it is not a far-fetched possibility that this RARRES1 fragment may act as a messenger for intercellular crosstalk for the development of glomerulopathy and chronic kidney disease. Also, it would be interesting to know whether this fragment is detectable in urine and whether it can be a biomarker for related renal diseases. Obviously, there is much more to learn about the regulation of RARRES1. Regardless, the discovery of the molecular processing of RARRES1 opens a new door to the understanding of RA signaling and the design of efficacious therapeutic strategies.

\section{Acknowledgments}

The authors were supported in part by the grants from the Department of Veterans Affairs (Merit Review Award I01 BX000319) and the NIH (DK058831, DK087843). ZD is a recipient of a Senior Research Career Scientist award from the Department of Veterans Affairs.

Address correspondence to: Zheng Dong, 1460 Laney Walker Boulevard, CB1124, Department of Cellular Biology and Anatomy, Medical College of Georgia at Augusta University, Augusta, Georgia 30912, USA. Phone: 706.721.2825; Email: zdong@augusta.edu.

1. Ghyselinck NB, Duester G. Retinoic acid signaling pathways. Development. 2019;146(13):dev167502.

2. Pawlikowski B, Wragge J, Siegenthaler JA. Retinoic acid signaling in vascular development. Genesis. 2019;57(7-8):e23287.

3. Marquez HA, Chen F. Retinoic acid signaling and development of the respiratory system. Subcell Biochem. 2020;95:151-174.

4. Mezquita B, Mezquita C. Two opposing faces of retinoic acid: induction of stemness or induction of differentiation depending on cell-type. Biomolecules. 2019;9(10):567.

5. Rosselot C, et al. Non-cell-autonomous retinoid signaling is crucial for renal development. Development. 2010;137(2):283-292.

6. Chiba $\mathrm{T}$, et al. Retinoic acid signaling coordinates macrophage-dependent injury and repair after AKI. J Am Soc Nephrol. 2016;27(2):495-508.

7. Yago-Ibáñez J, García-Pastor C, Lucio-Cazaña FJ, Fernández-Martínez AB. Retinoic acid receptor-beta prevents cisplatin-induced proximal tubular cell death. Biochim Biophys Acta Mol Basis Dis. 2020;1866(7):165795.

8. Yucel C, et al. All-trans retinoic acid prevents cisplatin-induced nephrotoxicity in rats. Naunyn Schmiedebergs Arch Pharmacol. 2019;392(2):159-164.

9. $\mathrm{Wu}$ J, et al. Retinoic acid attenuates contrastinduced acute kidney injury in a miniature pig model. Biochem Biophys Res Commun. 2019;512(2):163-169.

10. Balasubramanian S, Jansen M, Valerius MT, Humphreys BD, Strom TB. Orphan nuclear receptor Nur77 promotes acute kidney injury and renal epithelial apoptosis. J Am Soc Nephrol. 2012;23(4):674-686.

11. Nakamura J, et al. Myofibroblasts acquire 
retinoic acid-producing ability during fibroblast-to-myofibroblast transition following kidney injury. Kidney Int. 2019;95(3):526-539.

12. Dai Y, et al. Retinoic acid improves nephrotoxic serum-induced glomerulonephritis through activation of podocyte retinoic acid receptor $\alpha$. Kidney Int. 2017;92(6):1444-1457.

13. Peired A, et al. Proteinuria impairs podocyte regeneration by sequestering retinoic acid. JAm Soc Nephrol. 2013;24(11):1756-1768.

14. Li X, Dai Y, Chuang PY, He JC. Induction of retinol dehydrogenase 9 expression in podocytes attenuates kidney injury. J Am Soc Nephrol. 2014;25(9):1933-1941.

15. Datta PK, Lianos EA. Retinoic acids inhibit inducible nitric oxide synthase expression in mesangial cells. Kidney Int . 1999;56(2):486-493.

16. Mallipattu SK, et al. Kruppel-like factor 15 (KLF15) is a key regulator of podocyte differenti- ation. J Biol Chem. 2012;287(23):19122-19135.

17. Tamaki M, et al. All-trans retinoic acid suppresses bone morphogenetic protein 4 in mouse diabetic nephropathy through a unique retinoic acid response element. Am J Physiol Endocrinol Metab. 2019;316(3):E418-E431.

18. Sierra-Mondragon E, et al. All-trans retinoic acid attenuates fibrotic processes by downregulating TGF- $\beta 1 /$ Smad3 in early diabetic nephropathy. Biomolecules. 2019;9(10):E525.

19. Chen X, et al. The potential role of retinoic acid receptor $\alpha$ on glomerulosclerosis in rats and podocytes injury is associated with the induction of MMP2 and MMP9. Acta Biochim Biophys Sin (Shanghai). 2017;49(8):669-679.

20. He JC, et al. Retinoic acid inhibits HIV-1-induced podocyte proliferation through the cAMP pathway. JAm Soc Nephrol. 2007;18(1):93-102.

21. Xu Q, Lucio-Cazana J, Kitamura M, Ruan X,
Fine LG, Norman JT. Retinoids in nephrology: promises and pitfalls. Kidney Int. 2004;66(6):2119-2131.

22. Mallipattu SK, He JC. The beneficial role of retinoids in glomerular disease. Front Med (Lausanne). 2015;2:16

23. Elsayed AM, Abdelghany TM, Akool el-S, AbdelAziz AA, Abdel-Bakky MS. All-trans retinoic acid potentiates cisplatin-induced kidney injury in rats: impact of retinoic acid signaling pathway. Naunyn Schmiedebergs Arch Pharmacol. 2016;389(3):327-337.

24. Chen A, et al. Soluble RARRES1 induces podocyte apoptosis to promote glomerular disease progression. J Clin Invest. 2020;130(10):5523-5535.

25. Dimke H, Maezawa Y, Quaggin SE. Crosstalk in glomerular injury and repair. Curr Opin Nephrol Hypertens. 2015;24(3):231-238. 